\title{
Reabilitação com implante dentário em região atrófica de mandíbula através da técnica de Split Crest: relato de caso
}

Rehabilitation with dental implant in an atrophic region of the mandible using the Split Crest

technique: case report

Rehabilitación con un implante dental en una región atrófica de la mandíbula mediante la técnica

Caroline Liberato Marchiolli

ORCID: https://orcid.org/0000-0001-8881-4882 Universidade Brasil, Brasil

E-mail: caaliberato@gmail.com

Jéssica Monique Lopes Moreno

ORCID: https://orcid.org/0000-0002-8808-4872 Universidade Estadual Paulista "Júlio de Mesquita Filho", Brasil E-mail: jessica_morenoo@hotmail

Ricardo Junior Denardi

ORCID: https://orcid.org/0000-0001-8312-7493

Universidade de São Paulo, Brasil

E-mail:ricardojdenardi@gmail.com

Caroline Martin Denardi

ORCID: https://orcid.org/0000-0003-2003-1712

Centro Universitário Avantis, Brasil

E-mail: cmartindenardi@gmail.com

Wirley Gonçalves Assunção

ORCID: https://orcid.org/0000-0002-8903-0737

Universidade Estadual Paulista "Júlio de Mesquita Filho", Brasil

E-mail: wirley.assuncao@unesp

Jennifer Lorrayne Bezerra Araujo Recalde

ORCID: https://orcid.org/0000-0002-3066-5703

Centro Universitário Avantis, Brasil

E-mail: jennifer.akrieger@yahoo.com

Ingrid Kely dos Reis Santos

ORCID: https://orcid.org/0000-0001-7726-1792

Centro Universitário Avantis, Brasil

Email:ingrrid 21@hotmail.com

Ricardo Alves Toscano

ORCID: https://orcid.org/0000-0002-6381-463X

Universidade Estadual Paulista "Júlio de Mesquita Filho", Brasil

E-mail: odontosc.joi@terra.com.br

\begin{abstract}
Resumo
Introdução: A perda de volume alveolar é uma implicação clinica decorrente de extrações dentarias, que trazem comprometimento potencialmente importantes nos resultados estéticos e funcionais das reabilitações implanto suportada. O tratamento cirúrgico está indicado nos casos de pacientes com presença de acidente anatômico importante quanto na quantidade e qualidade do osso remanescente que apresentam envolvimento em espessura. Objetivo: O objetivo deste trabalho é relatar um caso clinico sobre reabilitação com implante dentário através da técnica de Split crest. Relato de caso: Paciente gênero feminino, 37 anos apresentava como queixa principal a ausência do elemento dental 35. Após anamnese, exame clinico e avaliação tomográfica da região de interesse observou -se o tecido ósseo remanescente com presença de acidente anatômico importante quanto ao volume vestibular. Após apresentação de alternativas de tratamento optou-se pela expansão de crista óssea pela técnica de Split crest e instalação imediata do implante. A técnica Split crest em mandíbula é uma técnica previsível para expansão de rebordos atróficos. A técnica mostrou-se viável propiciando um aumento significativo na espessura do rebordo, ausência de complicações e diminuição do tempo do tratamento ao paciente. Considerações finais: A permissão de instalação do implante juntamente com a expansão do rebordo se faz a maior vantagem da utilização da técnica corrigindo implicações clinicas potencialmente importante no resultado estético e funcional de reabilitações implanto suportado.
\end{abstract}

Palavras-chave: Reabilitação atrófica; Split Crest; Implante dentário. 


\begin{abstract}
Introduction: The loss of alveolar volume is a clinical implication resulting from dental extractions, which bring potentially important compromises in the aesthetic and functional results of implant-supported rehabilitations. Surgical treatment is indicated in cases of patients with the presence of an important anatomical accident as to the quantity and quality of the remaining bone that is involved in thickness. Objective: The aim of this paper is to report a clinical case of rehabilitation with a dental implant using the Split crest technique. Case Report: A 37 year-old female patient presented as main complaint the absence of dental element 35. After anamnesis, clinical examination and tomographic evaluation of the region of interest it was observed the remaining bone tissue with presence of important anatomical accident regarding the buccal volume. After presentation of treatment alternatives, we opted for bone crest expansion using the Split crest technique and immediate implant installation. The Split crest technique in the mandible is a predictable technique for expanding atrophic ridges. The technique proved to be feasible, providing a significant increase in ridge thickness, absence of complications and reduced treatment time for the patient. Final considerations: Allowing implant installation together with ridge expansion is the major advantage of using the technique to correct potentially important clinical implications in the aesthetic and functional outcome of implant-supported rehabilitations. Keywords: Rehabilitation, Atrophic; Split Crest; Implant dental.
\end{abstract}

\title{
Resumen
}

Introducción: La pérdida de volumen alveolar es una implicación clínica decadente de las extracciones dentales, que conlleva un compromiso potencialmente importante en los resultados estéticos y funcionales de las rehabilitaciones implantadas. El tratamiento quirúrgico está indicado en los casos de pacientes con presencia de un accidente anatómico importante en cuanto a la cantidad y calidad del hueso restante que está implicado en el espesor. Objetivo: El objetivo de este estudio es reportar un caso clínico de rehabilitación con un implante dental utilizando la técnica de la cresta Split. Reporte de caso: Paciente femenina, de 37 años, presentó como queja principal la ausencia del elemento dental 35. Después de la anamnesis, el examen clínico y la evaluación tomográfica de la región de interés se observó el tejido óseo remanente con presencia de accidente anatómico importante respecto al volumen bucal. Tras la presentación de las alternativas de tratamiento se optó por la expansión de la cresta ósea mediante la técnica de la cresta dividida y la instalación inmediata del implante. La técnica de la cresta dividida en la mandíbula es una técnica predecible para expandir las crestas atróficas. La técnica demostró ser viable, proporcionando un aumento significativo del grosor de la cresta, ausencia de complicaciones y reducción del tiempo de tratamiento para el paciente. Consideraciones finales: Permitir la instalación de implantes junto con la expansión de la cresta es la mayor ventaja de utilizar la técnica para corregir implicaciones clínicas potencialmente importantes en el resultado estético y funcional de las rehabilitaciones soportadas por implantes.

Palabras clave: Rehabilitación, atrófica; Cresta partida; Implante dental.

\section{Introdução}

Quantidades ósseas insuficientes comprometem o plano de tratamento na reabilitação com implantes dentários. Várias modalidades de tratamento são propostas para recuperar o tecido ósseo incluindo enxertos em bloco "Onlay", regeneração óssea guiada, distração osteogênica e densificação óssea.

Em 1986, Nentwig relatou uma alternativa para o aumento ósseo através de uma técnica de divisão da crista óssea que permitiu simultaneamente a expansão da crista alveolar e a inserção do implante dentário. A técnica consiste na divisão e expansão entre as tábuas ósseas vestibulares e lingual/palatinas. A placa cortical vestibular, através da expansão, é reposicionada lateralmente. Através dessa técnica será criado um espaço vazio que poderá ser ocupado por um implante dentário. Os espaços vazios remanescentes após instalação do implante podem ser preenchidos por biomateriais particulados associados ou não a agregados plaquetários. A osteotomia pode ser realizada convencionalmente através de fresas e brocas. A adoção de instrumentos rotatórios, oscilatório e ultra-sônicos permite uma osteotomia mais precisa com menor risco de trauma cirúrgico.

O grande benefício da técnica de Split Crest é a diminuição da quantidade de intervenções cirúrgicas, diminuição da morbidade e resolução mais rápida e eficaz da reabilitação implantossuportada em região com espessura óssea insuficiente. Para o sucesso na execução da técnica, há necessidade de uma quantidade mínima de tecido medular para permitir a elasticidade e a expansão das cristas ósseas. Cristas ósseas atróficas sem a presença de remanescente medular estão contra-indicadas para a técnica de expansão óssea. 
Revisão sistemática avaliou as taxas de sobrevivência e sucesso nos implantes dentários de titânio quando instalados em áreas submetidas a expansão da crista óssea. A taxa de sucesso global foi calculada em 96,6\% e a taxa de sobrevida foi de 96,8\% em um período médio de acompanhamento de 58,3 meses.

Um outro estudo de revisão sistemática e meta-análise avaliou a perda óssea mesial e distal do implante antes e após o carregamento protético em procedimento de expansão da crista óssea. Avaliou-se nesse estudo quantitativamente o ganho de volume ósseo. Vinte e sete estudos preencheram os critérios de seleção e foram incluídos na pesquisa. Observou-se um ganho efetivo médio de $3,8 \mathrm{~mm}$, independente dos instrumentos cirúrgicos utilizados na osteotomia, ou seja, os instrumentos piezoelétricos não apresentam vantagens em relação aos instrumentos convencionais para ganho ósseo. Autores concluíram que a técnica de Split Crest é efetiva e aumenta o volume do rebordo atrófico permitindo a instalação do implante com previsibilidade. A perda óssea ao redor dos implantes após a separação das paredes do tecido ósseo cortical foi semelhante à esperada quando os implantes são instalados em condições ideais.

\section{Relato de Caso}

Paciente do gênero feminino, sem comprometimento sistêmico (ASA I), 37 anos apresentou como queixa principal a ausência do elemento dental 35 (FDI). Após anamnese, exame clínico e avaliação tomográfica de feixe cônico (CBCT) da região de interesse observou-se um tecido ósseo remanescente com presença de acidente anatômico importante quanto ao volume vestibular, esta perda de volume alveolar que traz implicações clinicas potencialmente importantes no resultado estético final da reabilitação. (Figura 1). Havia necessidade da regeneração óssea da região para instalação do implante dentário. Clinicamente observou-se uma depressão óssea na face vestibular (Figura 2). Pelo corte transversal da tomografia pode ser observado um delgado tecido ósseo com presença de tecido ósseo medular. Após apresentação das alternativas de tratamento optou-se pela expansão de crista óssea e instalação imediata do implante dentário.

Figura 1 - CBCT pré-operatório.

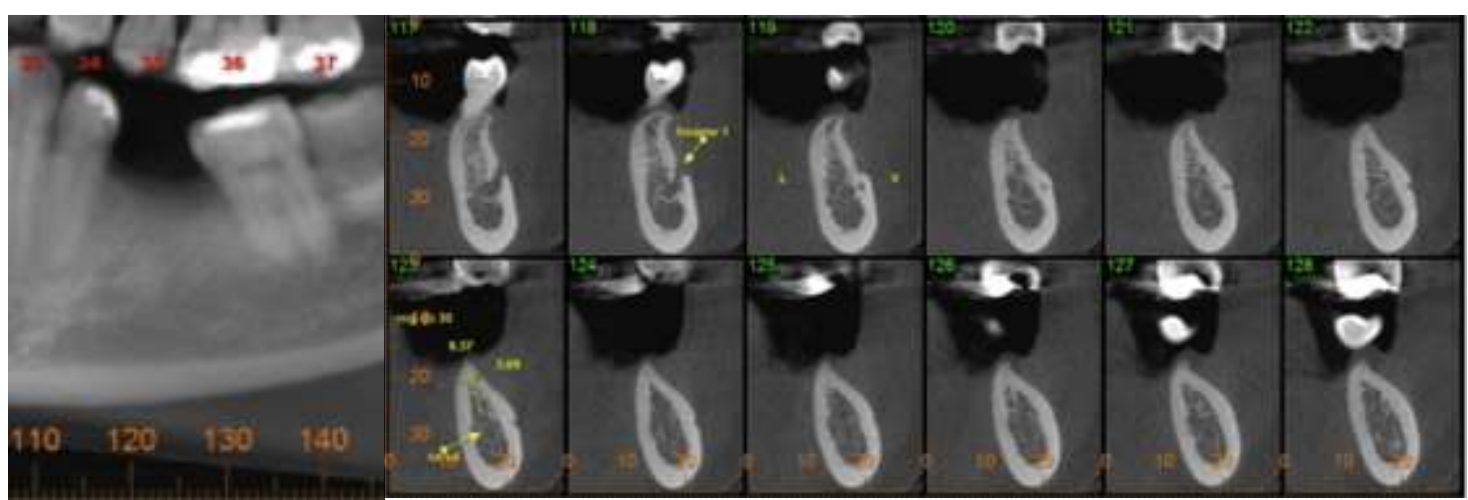

Fonte: Autores. 
Figura 2 - Aspecto clínico inicial de região de interesse.

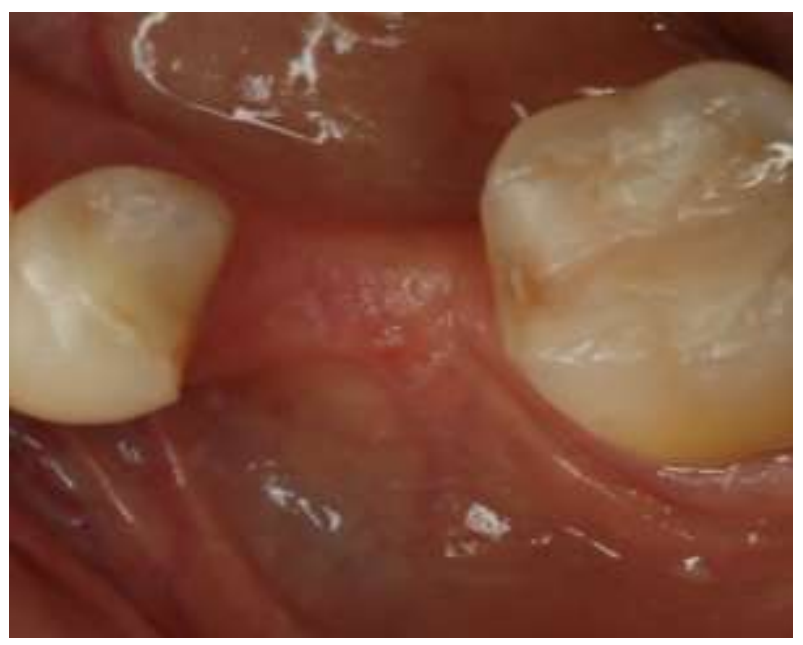

Fonte: Autores.

Foi realizada a venopunção para coleta do material sanguíneo para obtenção dos agregados plaquetários. $\mathrm{O}$ acesso venoso foi na veia cubital mediana com coleta a vácuo (Figura 3).

Figura 3 - Acesso para venopunção e coleta a vácuo do material sanguíneo

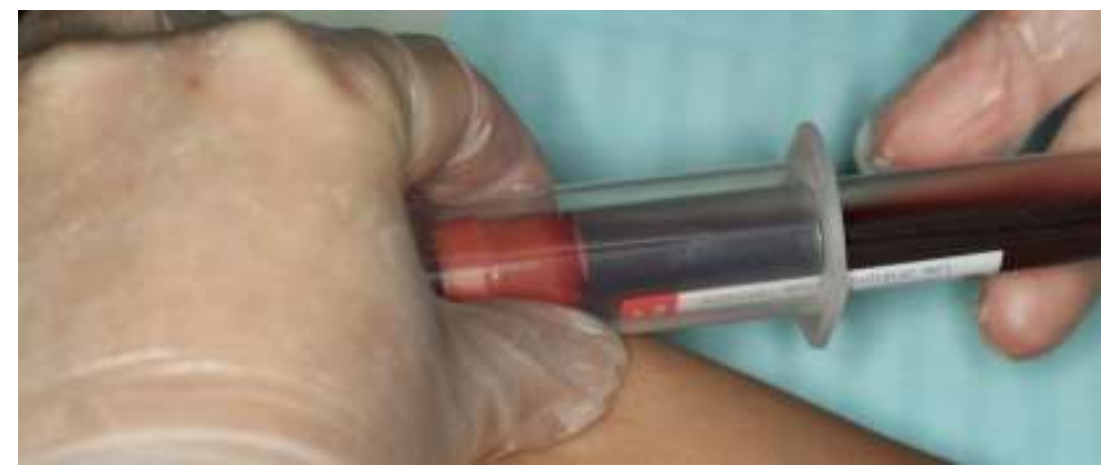

Fonte: Autores.

Foram coletados cinco tubos a vácuo com tampa vermelha de $9 \mathrm{ml}$ (Labor Import, Brasil). Os 4 tubos foram posicionados em centrífuga Kasvi digital 8x15 ml rotor de ângulo fixo 4000 rpm (Kasvi São jose dos Pinhais Parana Pr Brasil) digital de rotor fixo e centrifugados por 13 minutos a 2700rpm. Após centrifugação os tubos vermelhos foram retirados da centrífuga a fibrina foi removida do tubo e separada do restante do material sanguíneo com auxílio de uma tesoura e pinça metálica (Figura 4). 
Figura 4 - Obtenção da fibrina rica em plaquetas e leucócitos (L-PRF)

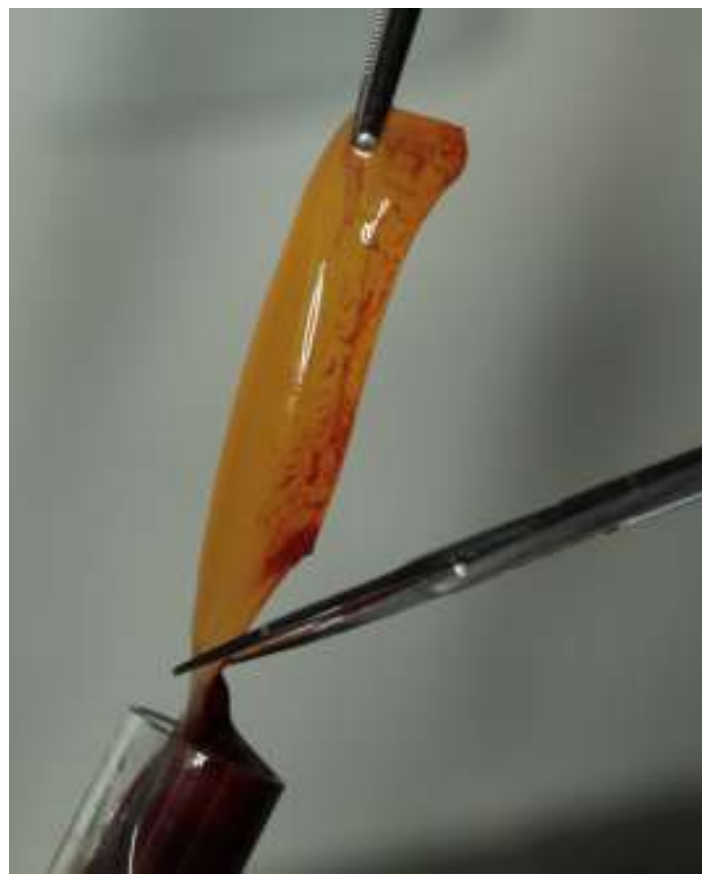

Fonte: Autores.

As fibrinas foram posicionadas sobre um box metálico para sua conformação em membranas de L-PRF (Figura 5).

Figura 5 - Fibrinas sobre o box metálico.

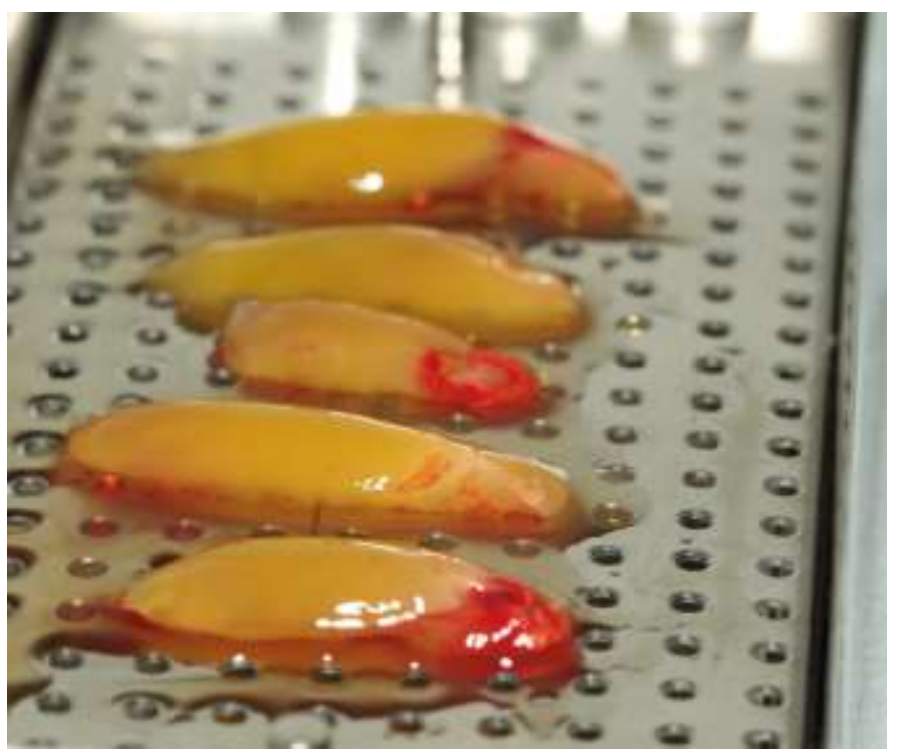

Fonte: Autores.

Na região da ausência do elemento dental condenado 35 (FDI) foi utilizada a técnica anestesia infiltrativa em fundo de vestíbulo com complementação na lingual utilizando anestésico injetável local à base de cloridrato de articaína com epinefrina 1:100.000 (DFL, Rio de Janeiro, RJ, Brasil). Após incisão intrasulcular nos elementos dentais adjacentes e incisão supracrestal na região edêntula foi realizado o descolamento total, remoção total do tecido expondo o remanescente ósseo existente preservando o máximo possível de periósteo aderido nas tabuas ósseas. (Figura 6). 
Figura 6 - Aspecto clínico após descolamento dos tecidos.

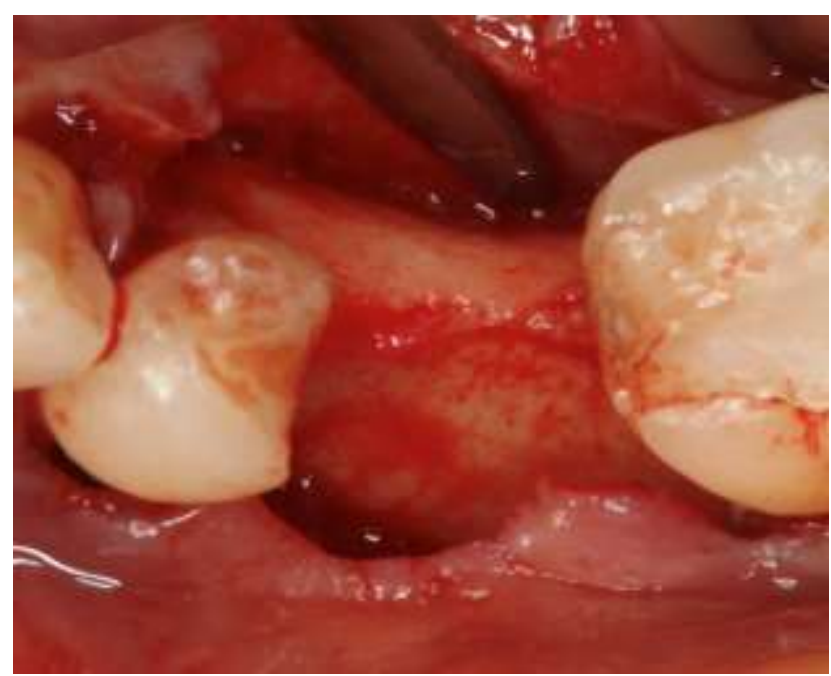

Fonte: Autores.

Inicialmente foi realizada uma osteotomia na crista óssea distante aproximadamente $3 \mathrm{~mm}$ dos dentes adjacentes. Na sequência, mantendo a mesma distância de $3 \mathrm{~mm}$ dos dentes adjacentes, foram realizadas duas osteotomias oblíquas na mesial e distal da face vestibular com utilização de pontas e equipamentos ultra-sônicos (CVDentUS ${ }^{\circledR}$, Brasil). A osteotomia supracrestal e as oblíquas tiveram como limite de profundidade o tecido ósseo medular (Figura 7).

Figura 7 - Osteomias supracrestal e oblíquas.

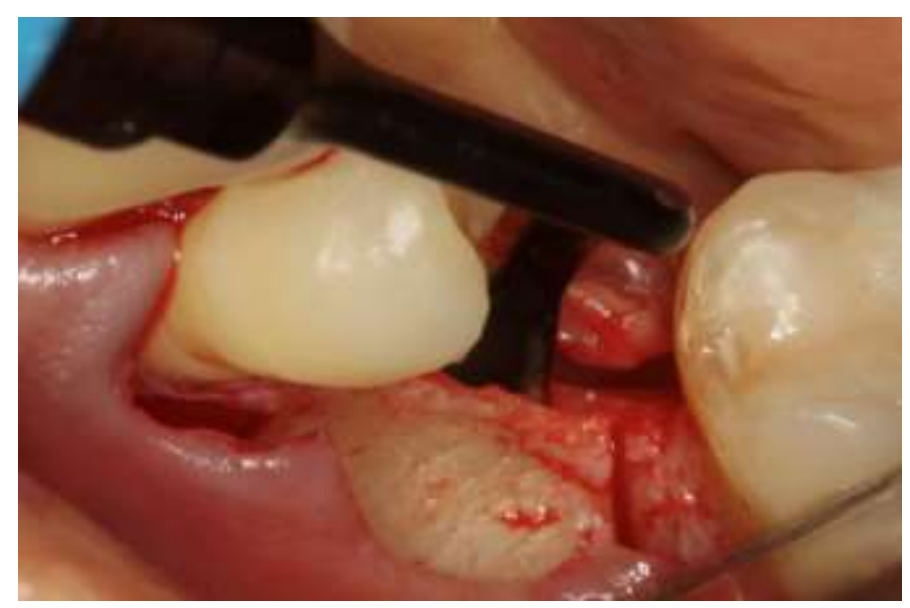

Fonte: Autores.

Após as osteotomias e com auxílio de martelo e cinzéis expansores iniciou-se a expansão das tábuas ósseas vestibular (Figura 8). O cinzel (Expansor Cinzel, DSP Biomedical ${ }^{\circledR}$, Campo Largo, Brasil) foi introduzido no remanescente ósseo até a profundidade de perfuração planejada previamente para instalação do implante dentário (9mm). Após a utilização do cinzel foi realizada a instrumentação com a fresa $2.0 \mathrm{~mm}$ até a profundidade de $9 \mathrm{~mm}$ (Figura 9). Na sequência os expansores escalonados com a catraca foram utilizados para auxiliar a expansão da tábua óssea vestibular (Kit Trenchfit, DSP Biomedical ${ }^{\circledR}$, Campo Largo, Brasil) até a profundidade de 9mm (Figura 10). Após utilização dos cinzéis e expansores observa-se clinicamente o afastamento lateral da tábua óssea vestibular (Figura 11). 
Research, Society and Development, v. 10, n. 11, e329101119116, 2021

(CC BY 4.0) | ISSN 2525-3409 | DOI: http://dx.doi.org/10.33448/rsd-v10i11.19116

Figura 8 - Utilização do cinzel para expansão das cristas.

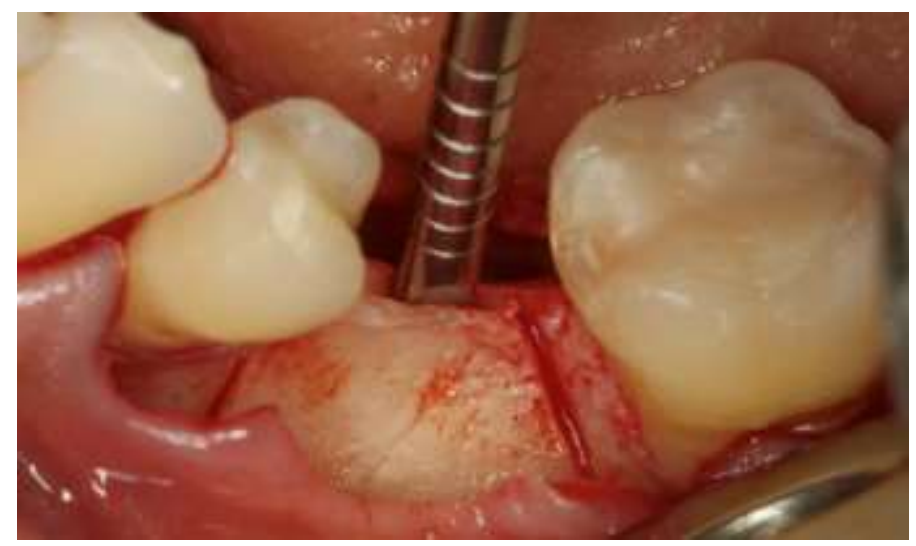

Fonte: Autores.

Figura 9 - Instrumentação com a fresa de diâmetro $2 \mathrm{~mm}$.

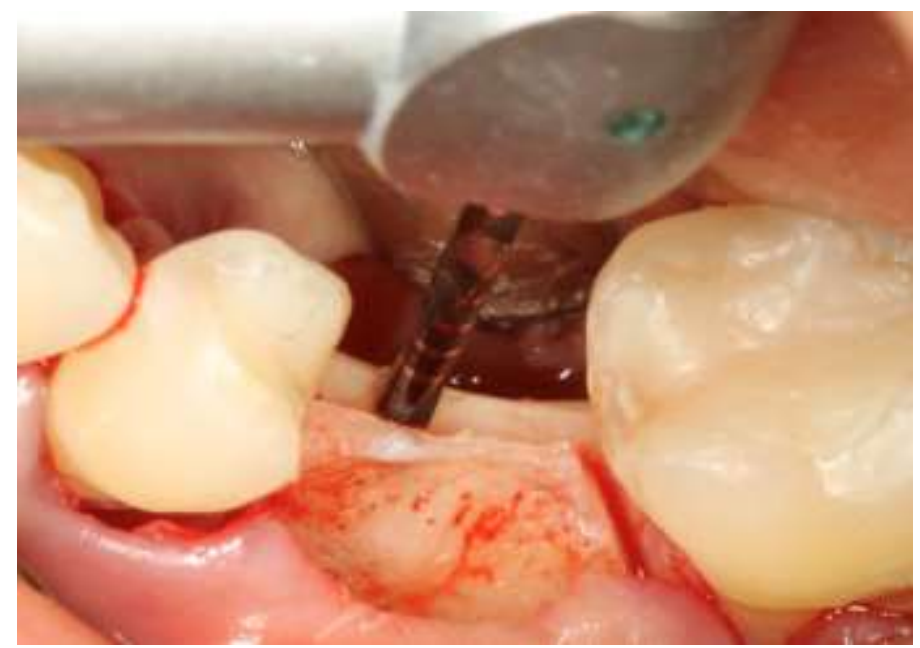

Fonte: Autores

Figura 10 - Utilização dos expansores de crista óssea de diâmetro 3,5 e 3,75mm.

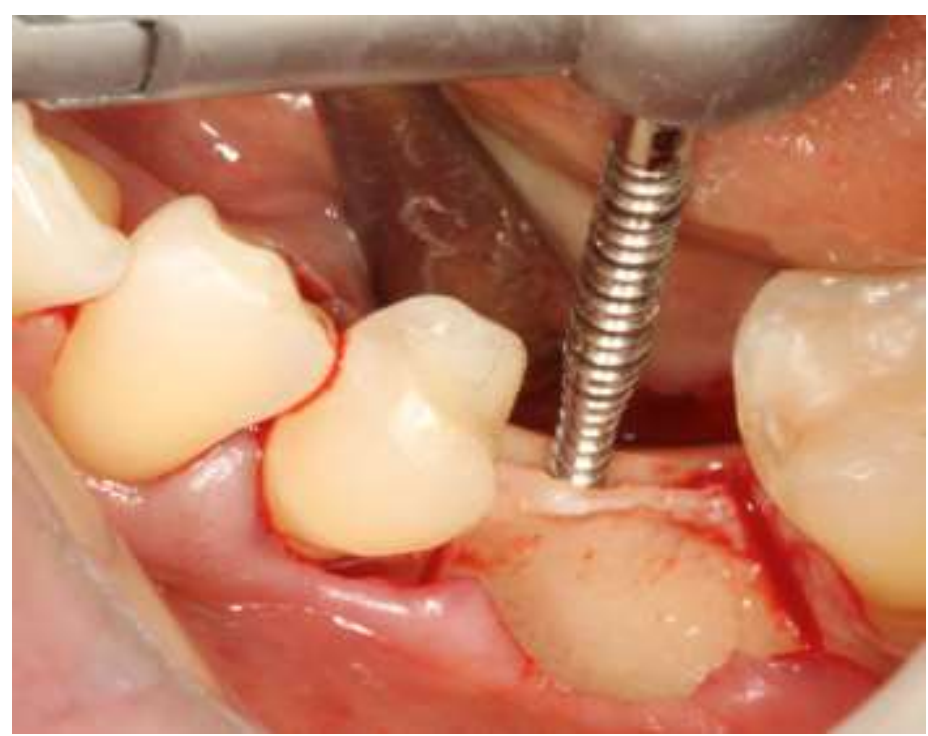

Fonte: Autores. 
Research, Society and Development, v. 10, n. 11, e329101119116, 2021

(CC BY 4.0) | ISSN 2525-3409 | DOI: http://dx.doi.org/10.33448/rsd-v10i11.19116

Figura 11 - Vista oclusal após expansão das cristas ósseas.

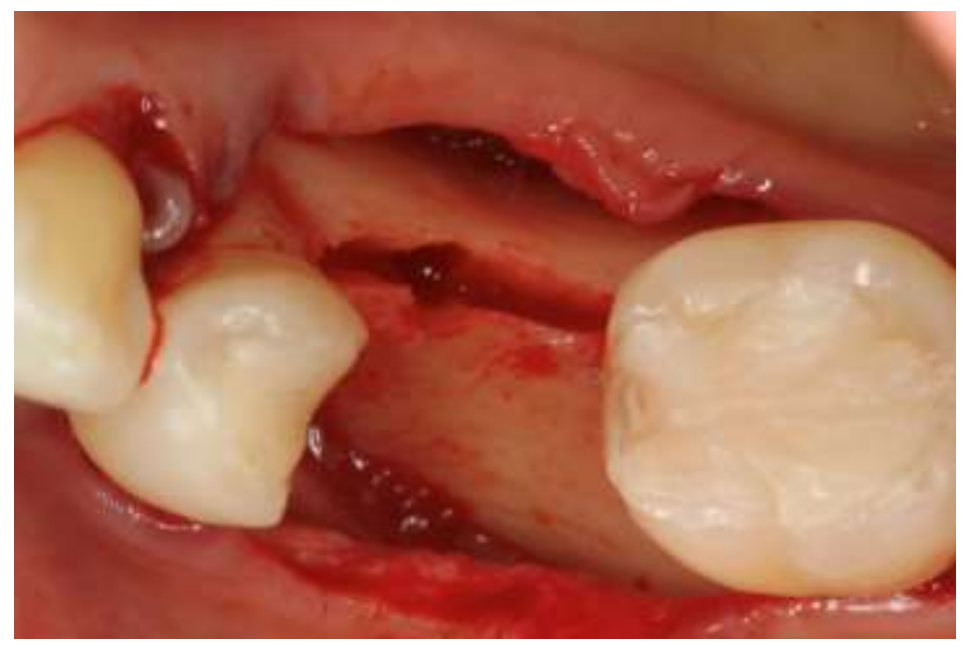

Fonte: Autores.

Após a expansão escalonada conforme o diâmetro e profundidade foi instalado implante Cone Morse Indexado Biofit 3,75x7mm (DSP Biomedical ${ }^{\circledR}$, Campo Largo, Brasil) (Figura 12).

Figura 12 - Vista oclusal após instalação do implante dentário.

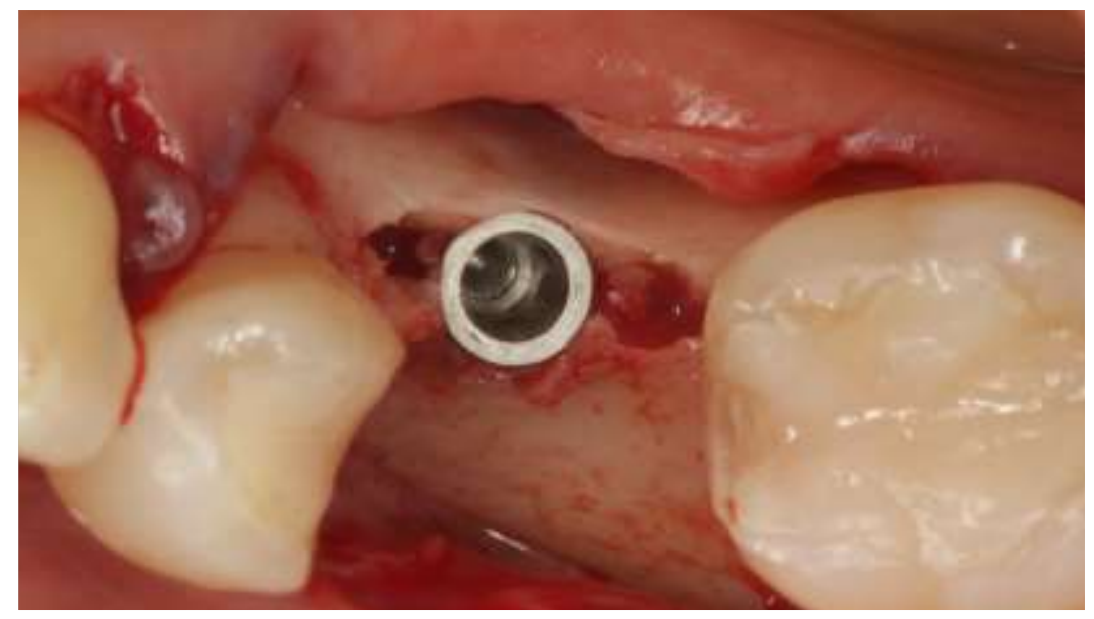

Fonte: Autores.

Os espaços vazios resultantes da expansão óssea foram preenchidos com biomaterial xenógeno Bio-Oss ${ }^{\circledR}$ Large (Geistlich Pharma AG, Wolhusen, Suíça) (Figura 13). 
Research, Society and Development, v. 10, n. 11, e329101119116, 2021

(CC BY 4.0) | ISSN 2525-3409 | DOI: http://dx.doi.org/10.33448/rsd-v10i11.19116

Figura 13 - Preenchiomento do espaço vazio com Bio-Oss ${ }^{\circledR}$.

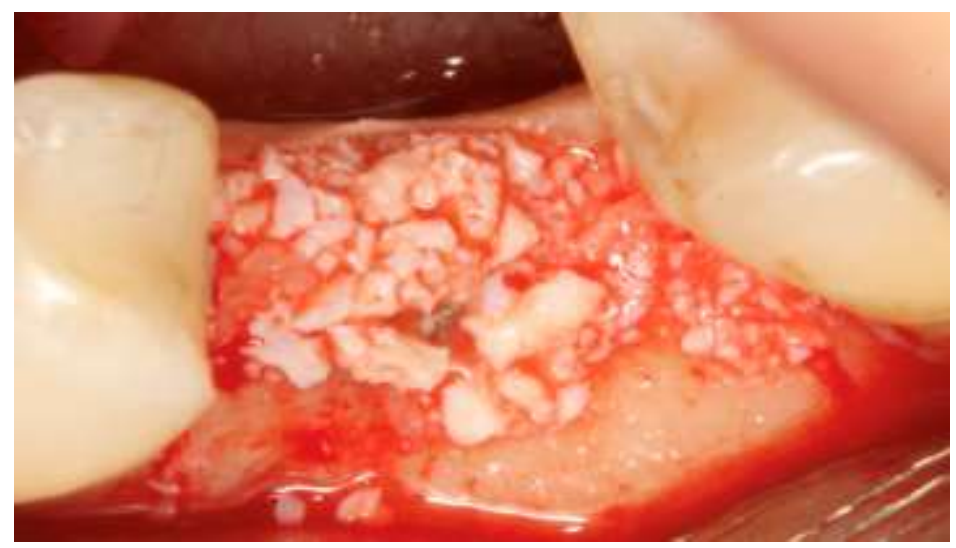

Fonte: Autores.

Sobre toda a área expandida foram posicionadas membranas de fibrina rica em plaquetas e leucócitos (L-PRF) (Figura 14).

Figura 14 - L-PRF aposicionada sobre a região expandida.

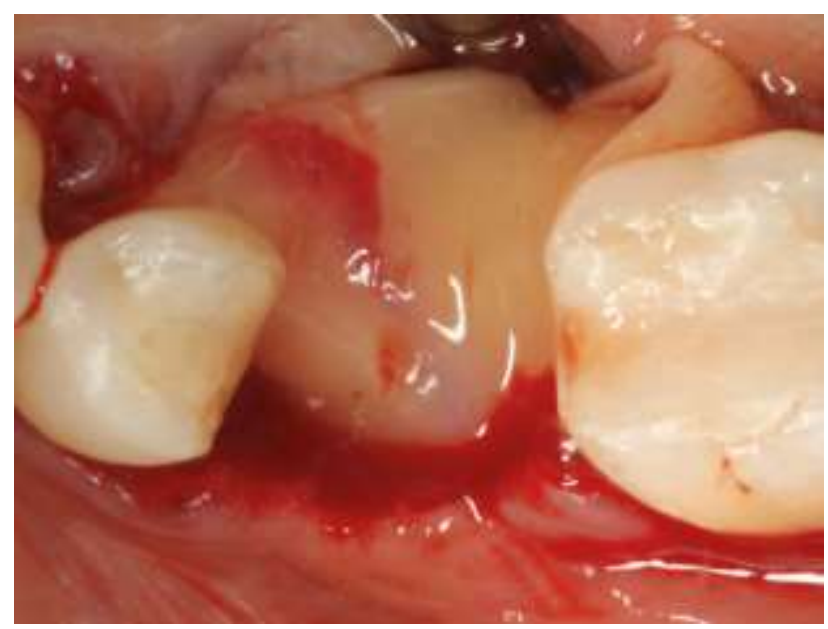

Fonte: Autores.

Após 6 meses de controle (Figura 15) observa-se uma estreita faixa de mucosa ceratinizada (Figura 16). 


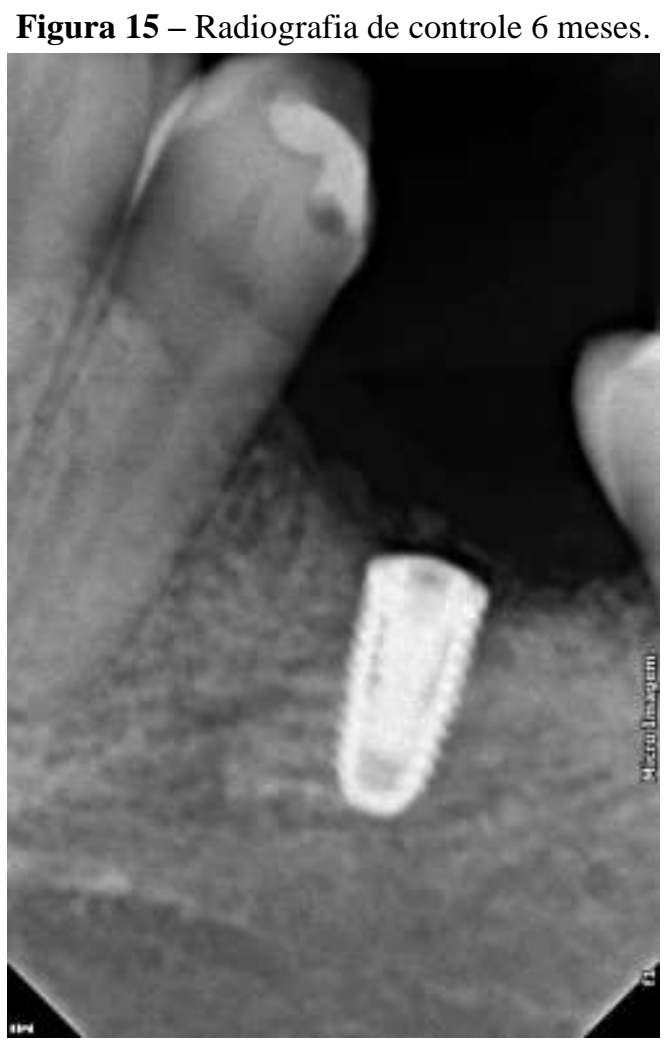

Fonte: Autores.

Figura 16 - Estreita faixa de mucosa ceratinizada.

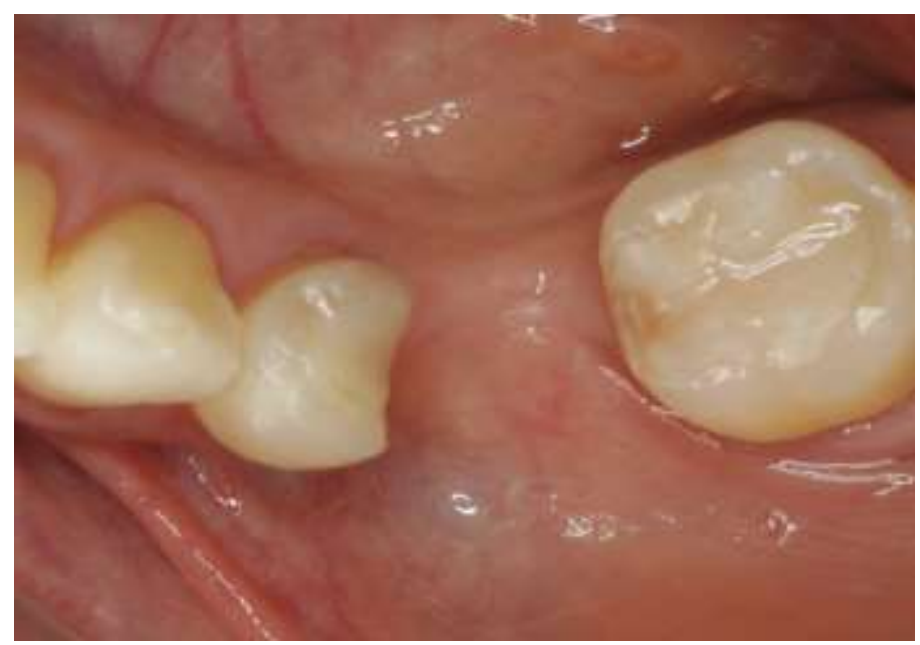

Fonte: Autores.

Para a cirurgia de reabertura foi realizada uma incisão com retalho divido próxima a linha mucogengival para a realização de enxerto gengival livre na região e consequente aumento da faixa de tecido ceratinizado (Figura 17). A região doadora foi a palatina. Para minimizar o desconforto pós-operatório na região doadora foi posicionado uma esponja hemostática de colágeno (Hemospon, Maquira, Maringá, Brasil) (Figura 18). O enxerto gengival livre foi estabilizado no leito receptor através de suturas de estabilização no periósteo. No mesmo momento do enxerto gengival livre o tapa implante foi substituído por uma cicatrizador (Figura 19). Após 60 dias observa-se a estabilidade e aumento da faixa de mucosa ceratinizada. O Intermediário protético pilar flexcone altura 2,5mm (DSP Biomedical ${ }^{\circledR}$, Campo Largo, Brasil) foi instalado com torque de 30N.cm (Figura 20). 
A coroa metalocerâmica foi confeccionada e instalada para reabilitação implanto-suportada na região (Figura 21). Oito meses após o procedimento observa-se a estabilidade peri-implantar na região operada (Figura 22).

Figura 17 - Retalho dividido próximo a linha mucogengival

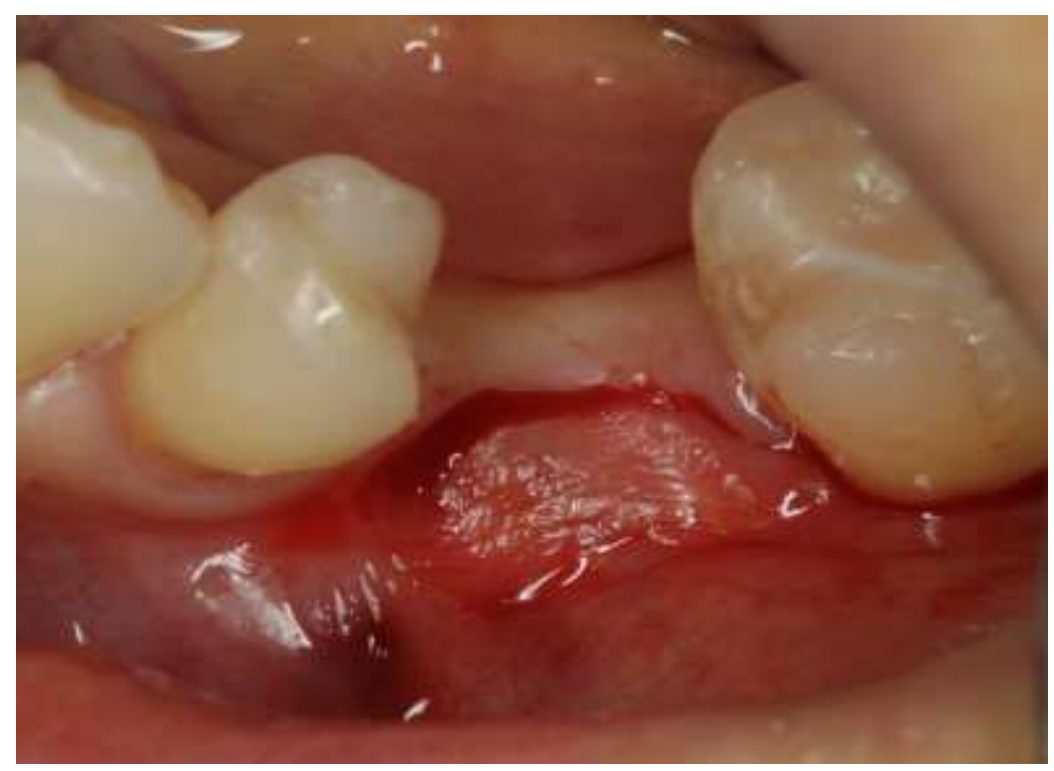

Fonte: Autores.

Figura 18 - Esponja hemostática de colágeno estabilizada na região doadora.

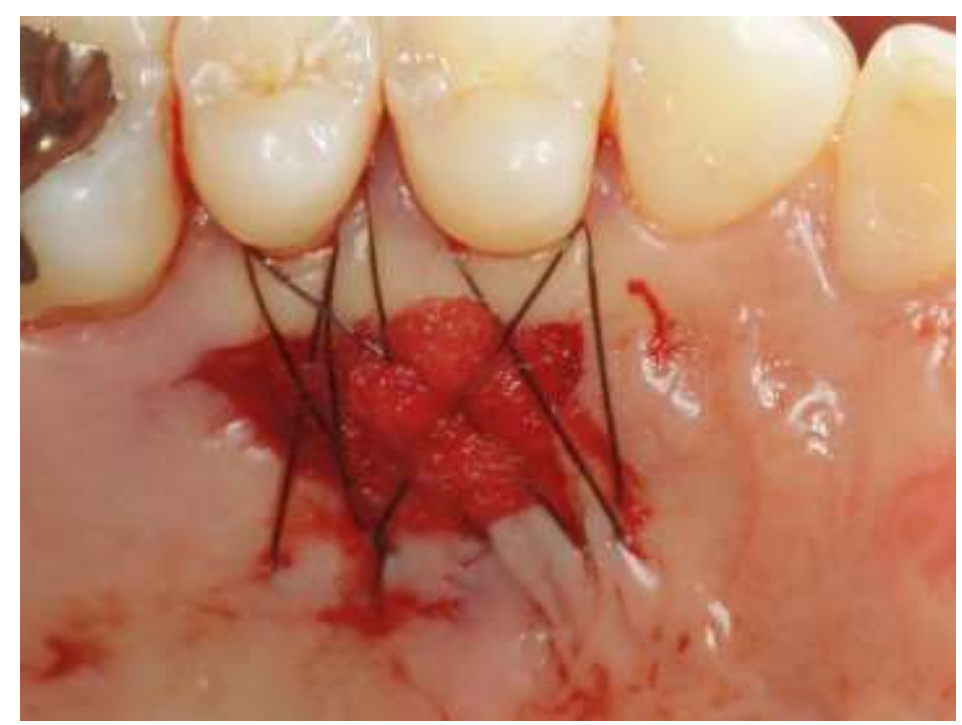

Fonte: Fonte: Autores. 
Research, Society and Development, v. 10, n. 11, e329101119116, 2021

(CC BY 4.0) | ISSN 2525-3409 | DOI: http://dx.doi.org/10.33448/rsd-v10i11.19116

Figura 19 - Enxerto gengival livre estabilizado na região receptora.

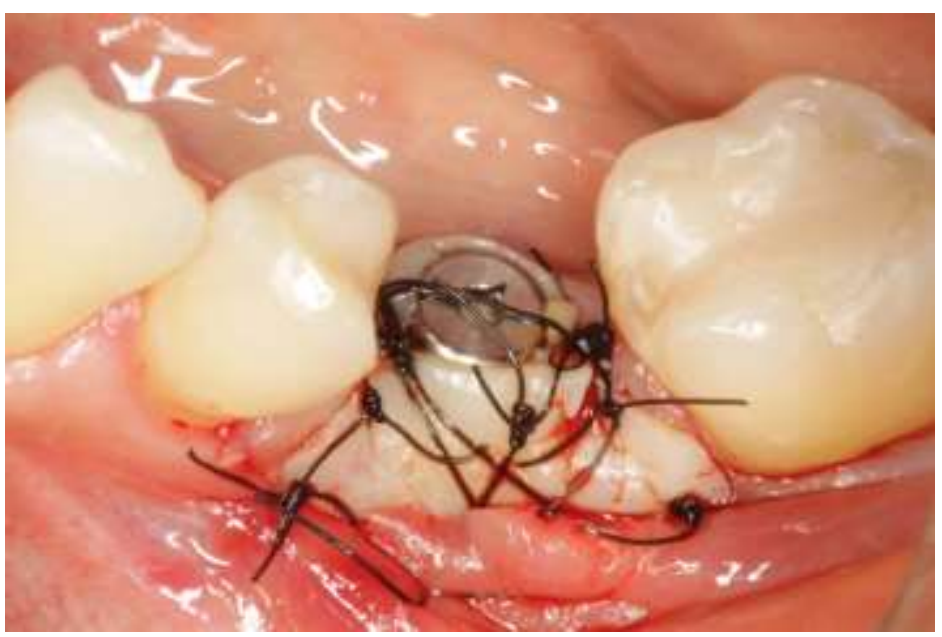

Fonte: Autores.

Figura 20 - Intermediário Flexcone instalado sobre o implante.

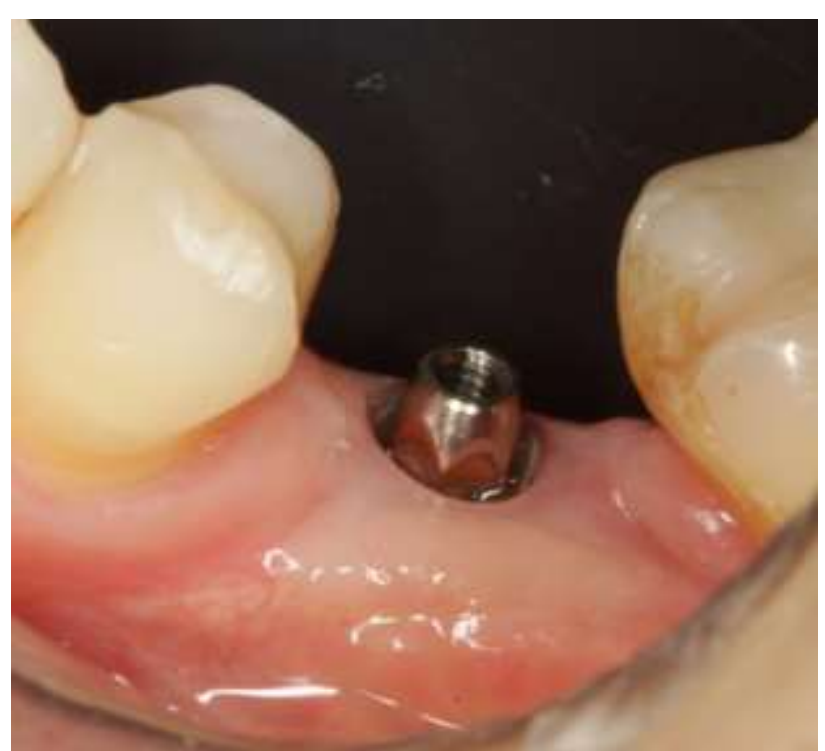

Fonte: Autores. 
Figura 21 - Reabilitação com coroa metalocerâmica.

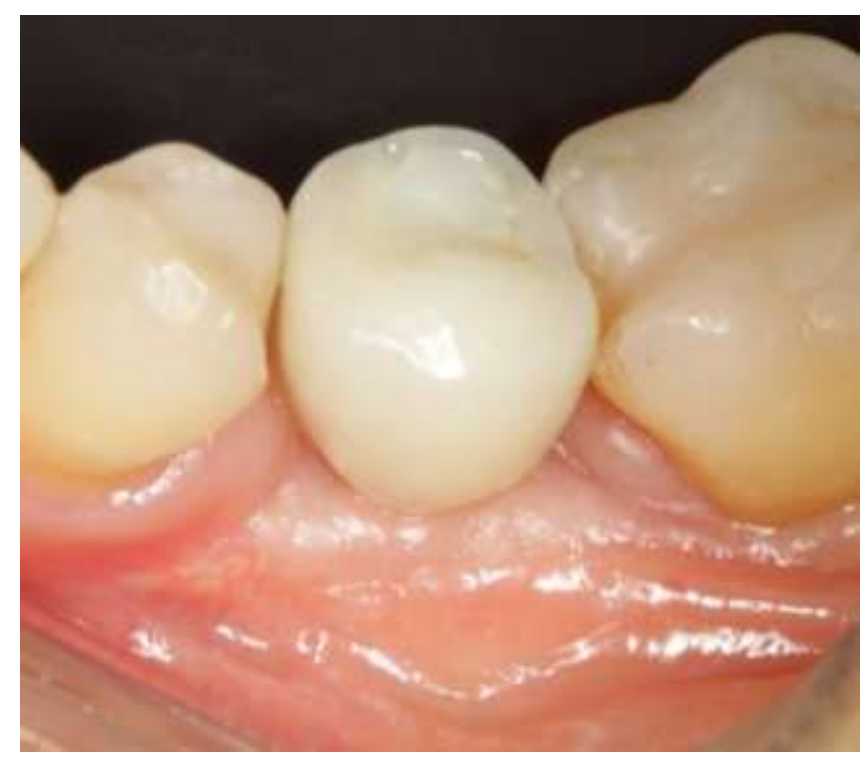

Fonte: Autores.

Figura 22 - Radiografia periapical de acompanhamento.

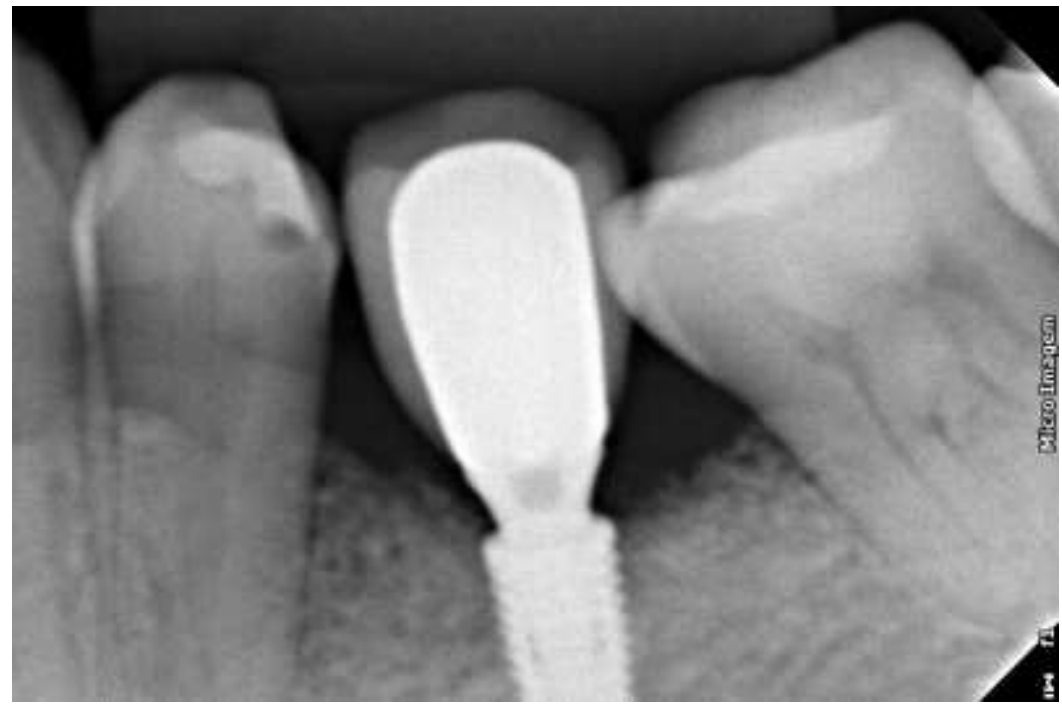

Fonte: Autores.

\section{Considerações Finais}

A técnica split crest em mandíbula, é uma técnica previsível para expansão de rebordos atróficos. A técnica mostrou-se viável propiciando um aumento significativo na espessura do rebordo, ausência de complicações diminuição do tempo do tratamento ao paciente. A permissão de instalação do implante juntamente com a expansão do rebordo se faz a maior vantagem da utilização da técnica corrigindo implicações clinicas potencialmente importante no resultado estético e funcional de reabilitações implanto suportado. 


\section{Referências}

Anitua, E., Begona, L., \& Orive, G. (2011) Two-stage split-crest technique with ultrasonic bone surgery for controlled ridge expansion: a novel modified technique. Oral Surg Oral Med Oral Pathol Oral Radiol Endod 112:708-10.

Blus, C., \& Szmukler-Moncler, S. (2006) Split-crest and immediate implant placement with ultra-sonic bone surgery: A 3-year life-table analysis with 230 treated sites. Clin Oral Implants Res 17:700-707.

Blus, C., Szumukler-Moncler, S., Vozza, L., Rispoli, L., \& Polastri, C. (2010), Split- crest and immediate implant placement with ultra-sonic bone Surgery (piezosurgery): 3-year life-table analysis with 180 treated sites. Quintessence Int 41:463-469.

Bravi, F., Bruschi, G. B., \& Ferrini, F. (2007), A 10-year multicenter retrospective clinical study of 1,715 implants placed with the edentulous ridge expansion technique. Int J Periodontics Restorative Dent 27:557-565.

Chiapasco M., Ferrini, F., Casentini, P., Accardi, S., \& Zaniboni, M. (2006), Dental implants placed in expanded narrow edentulous ridges with the Extension Crest device. A 1-3-year multicenter follow-up study. Clin Oral Implants Res 17:265-272.

Crespi, R., Capparè, P., \& Gherlone, E. F. (2014), Electrical mallet provides essential advantages in split-crest and immediate implant placement. J Oral Maxillofac Surg 18:59-64.

Mestas, G., Alarcón, M., \& Chambrone, L. (2016) Long-Term Survival Rates of Titanium Implants Placed in Expanded Alveolar Ridges Using Split Crest Procedures: A Systematic Review. Int J Oral Maxillofac Implants. 31(3):591-9. 10.11607/jomi.4453.

Montero, J., López-Velarde, A., \& de Diego, R. G. (2012) A retrospective study of the risk factors for ridge expansion with self-tapping osteotimes in dental implant surgery. Int J Oral Maxillofac Implants 27:203-210.

Nentwig, G. H. Technic of bone splitting for alveolar recession in anterior maxillary region. Quintessenz 1986, 37:1825-34.

Rahpeyma, A., Khajehahmadi, S., \& Hosseini, V. R. Lateral ridge split and immediate implant placement in moderately resorbed alveolar ridges: how much is the added width. Dent Res J (2013), 10:602-8.

Sakamoto, Y., Takasu, R., Hongo, T., Harada, N., Hasegawa, Y., \& Tsuyama, Y. (2011) The conservative surgical strategy for insufficient alveolar ridge employing split crest and socket lift procedure. Int J Oral Maxillofac Surg 40:1047.

Tolstunov, L., \& Hicke, B. (2013) Horizontal augmentation through the ridge-split procedure: a predictable surgical modality in implant reconstruction. $J$ Oral Implantol 39:

Waechter, J., Leite, F. R., Nascimento, G. G., Carmo Filho, L. C., \& Faot, F. (2017) The split crest technique and dental implants: a systematic review and metaanalysis. Int J Oral Maxillofac Surg. 46(1):116-128. 10.1016/j.ijom.2016.08.017. 